excellent summary of the more common tumours presenting to the surgeon and their appropriate form of management. Whilst not aiming to give the surgical detail of a Wipple resection, some very useful key points to bear in mind whilst undertaking this demanding operation are stressed. I was surprised to read the recommendation of temporary biliary drainage prior to undertaking this operation in jaundiced patients, as there is no evidence to recommend this practice routinely. Endocrine and cystic tumours of the pancreas and

The management of gastric cancer is well summarised including the results of recent chemotherapy trials, but the detailed surgical techniques and their indications were by necessity omitted. Colorectal cancer surgery has traditionally been the domain of all general surgeons, but with the advent of pouch surgery and more sphincter-preserving operations has come an even greater need for specialist colorectal surgeons. The issue of population screening for colorectal cancer is well covered, as is the detection of liver metastases, although I disagreed with the recommendation that their presence or absence should be established pre-operatively in all cases. The surgical treatment of carcinoma of the rectum is given a separate section although there is no mention of transanal excision, resection or irradiation and carcinoma of the anal canal is not discussed. The management of hepatic metastases from colorectal cancer is controversial with the place of surgical resection, regional and systemic chemotherapy uncertain; unfortunately the section devoted to it does not clarify this contentious subject.

This book contains a good summary of the management of the more common endocrine tumours, but perhaps a section about the multiple endocrine neoplasia syndromes could have been included. The four chapters on urological oncology were of interest to me as a non-urologist, although possibly out of place in a book of this length and reading audience. Bone and soft tissue sarcomas are discussed together, with a bias towards the former which was not as one might expect in a book of this type. In the chapter on melanoma the importance of complete nodal clearance whenever this operation is performed for nodal disease is emphasised and whilst isolated limb perfusion is mentioned, there are no details of the technique or results of its use for recurrent disease.

It is in the management of breast cancer that the surgeon's role as a member of a multi-disciplinary team is probably best exemplified and the chapter related to this section gives a good basic overview. There is little information about the enormous advances in molecular biology and the use of prognostic factors. Surprisingly, breast reconstruction and the management of locally advanced disease are not discussed. The final sections on the principles of cancer chemotherapy and recent advances in cancer research provided succinct informative reviews of these interesting topics.

In conclusion, it is difficult to define the market at which this book is aimed, although it does succeed in its aim of providing an up to date review of the topics covered. This book lacks the detail necessary for the consultant general surgeon and the surgeon or senior registrar with a special interest in oncology. It is, however, a useful overview for those preparing for the second part FRCS, but is not, as is claimed by the publishers, essential reading.

N.P.M. Sacks gall bladder carcinoma are not covered.

to rectify this by reducing the tabular data in a variety of ways. A series of bar charts of incidence by cancer site is organised in sequences of highest to lowest rates, pie charts by country and selected cancer sites are given and fuller age specific incidence graphs are shown by site by country.

There is a brief chapter giving explanation details - which is adequate - but no further words of comment or explanation are given in the text.

Graphical data always have an attraction way beyond their tabular counterparts and this volume is very appealing. Its systematic approach makes this an immensely useful volume to turn to and it should provide material for comment, investigation and teaching throughout the world.

There is very little to criticise - the user should beware of the caveats regarding the accuracy of the companion volume - in particular beware of over interpretation of data for some registries where small case numbers occur and where good census data are not always readily available. The diagrams occasionally become victims of their own logical scheme in that some of the pie diagrams do not shade consistently. Such comments are trivial compared with the overwhelming burden of good and interesting data, well presented in a clear fashion and understandable to all.

Atlas for US Cancer Mortality Among Non-Whites: 19501980, L.W. Pickle, T.J. Mason, N. Howard, R. Hoover and J.F. Fraumeni, Washington: NIH Publications, 1990, 186 pp.

In many ways this atlas is a far less satisfactory product than the previous work. It is also a companion volume to a previous publication - in this case an atlas on cancer mortality in US white populations. The authors are thus constrained by decisions made about that volume in the production of this one. Non-whites means the black, amerindian and asian members of the community. Numbers of the latter two groups are small and the data are dominated by the black population. The descriptive elements are not full or critical enough for the reader to acquire very much sense of the interpretability of the results. The geographic analysis is somewhat confused by the use of 'state economic areas' which are not fully explained in the text.

Perhaps the most useful parts of the results appear in the early pages where comparison of rates with whites are made followed by changes in the non-white rates with time. These reveal recent higher rates of male lung cancer in non-whites and the better known excesses of myeloma, prostate and oral cavity cancers in certain groups. Similarly there are lower rates of Hodgkin's Disease and melanoma.

The maps - four for each sex by site - take some while to appreciate fully - giving rates for each of the 3 decades and a time trend. However, only the extremes of the distribution are coloured. Because the numbers of non-whites is not evenly distributed around the country, for me, this makes interpretation very difficult indeed. The authors draw attention to some aspects of the geography about which they are confident but it is difficult to see what else can be gleaned from this compilation.

This is thus very much a specialist volume - useful to certain epidemiologists, geographers and public health specialists in the US but it is unlikely to have much general appeal.

R.A. Cartwright

Patterns of Cancer in Five Continents, IARC Scientific Publication No. 102, edited by S.L. Whelan, D.M. Parker and E. Masuger, Lyon: WHO, 1990, 162 pp. $£ 25.00$.

This slim volume is, in effect, the tabular part of the latest Cancer Incidence in Five Continents (Vol. 5) published in 1987 which incorporates incidence data from 36 countries, for all malignancies for the years $1978-82$ or thereabouts. The plethora of tabulation in this volume make it difficult to digest, even for epidemiologists. The present book attempts
Cancer: Causes, Occurrence and Control (IARC Scientific Pubs. No. 100), edited by L. Tomatis (editor-in-chief), A. Aitio, N.E. Day, E. Heseltine, J. Kaldor, A.B. Miller, D.M. Parkin and E. Riboli, Lyon: IARC, 1990, 352 pp. $£ 19.00$.

Epidemiology and prevention together form the principal focus of the activities of the International Agency for Research on Cancer, the autonomous cancer research arm of WHO. It is appropriate therefore that it should have chosen 
to devote its 100th publication to a review of these subjects.

Early chapters of this book concern measuring the burden of cancer worldwide, and a summary (42 pages) of the descriptive epidemiology of cancers, site by site. A major section (135 pages) concerns aetiology, cause by cause including complex exposures, single agents, and also dietary, reproductive and socio-economic factors. A useful list (p 118-121, Table II) of carcinogenic exposures unfortunately requires attention to the errata if confusion is to be avoided. No less than 59 of these are considered as definite human carcinogens, 41 probable and 182 as possible. Three novel chapters deal with population screening and preventive measures for different cancers, site by site, including an attempt to quantify the effects of such measures.

The chapter on the meaning of cause stresses the preferred operational definition - any factor, the elimination of which decreases the occurrence of a disease in a population. Consistent with this definition, azathioprine is accepted as a definite cause of lymphomas (probably because the resulting immunosuppression facilitates expression of the underlying viral cause). On the other hand, the HIV viruses (like the Epstein Barr virus) qualify only as associations with, and not as causes of, cancer despite the more than 1,000-fold increase of Kaposi's sarcoma that AIDS has produced.

There is a wealth of information here. Some unevenness is understandable given its multi-authorship and wide scope, and minor inaccuracies have slipped through. Thus the reference quoted does not support the statement that breast cancer mortality in some British nuns is an exception to Ramazzini's well-known observation (p. 70).

The work is orientated towards the Agency's previous publications and access to these, particularly the important monograph series on the Evaluation of Carcinogenic Risks to Humans, is often necessary if the source reference is to be traced. This book can be recommended as a valuable summary of much of what is known about the causes and prevention of human cancer.

L.J. Kinlen

\section{Complications of Cancer Management}

P.N. Plowman, T. McElwain \& A. Meadows. Oxford: Butterworth-Heinemann, 1991. 504 pp. $£ 98.00$.

Oncologists, perhaps more than most health care professionals, are concerned with the complications of their treatments. This apparent self-flagellation is most important and not just an exhibition of masochism. There can be no doubt that our patients frequently are put at considerable risk by the therapy we prescribe for attempted cure or even palliation. This book is an excellent comprehensive review of all aspects of this theme which involves many disciplines. Surgery, radiotherapy, chemotherapy, haematology, paediatrics and psychiatry are all represented in the list of authoritative contributors.

The book has been planned with three separate themes represented by separate sections. These cover chapters on general aspects, site orientated complications and a rather orphan small section at the end of topics particularly not covered elsewhere. The book is uncompromising in its approach to the specialist reader. It opens immediately with a review of the complications of drug therapy and ends with one on how patients cope with cancer. Space is not wasted on the usual anodyne introductions and finale, an editorial policy which is both refreshing and acceptable.

Inevitably there is some variability in the standard of the 31 different chapters written by a total of 57 authors. There is also some repetition. For example four chapters give details of the linear quadratic equation approach to fractionated radiation doses. Two consecutive chapters detail with text, figures and table the haemopoietic cell progenitor hierarchy. These are small criticisms of what are facts of relevance to these individual chapters. As someone who has edited multi-author books the decision to leave such apparent redundancies is familiar and inevitable!

A book review of the subjects will inevitably sound like a regurgitation of the chapter contents but this reviewer makes no apology for it as it is necessary to indicate the range of subjects. There are general chapters on the complications of chemotherapy, radiotherapy and combined modality treatments. There is a very useful and comprehensive review of the carcinogenic and genotoxic effects of treatment. Other chapters are concerned with acute and late effects as specifically applied to children or adults and include such problems as fertility and orthopaedic complications.

The site specific section covers all the main organ systems usually with separate chapters for chemotherapy and radiotherapy. This formula is useful but does result in some repetition. In addition neuropsychological aspects are also reviewed.

The third section is a short one with four chapters on minimising and overcoming the effects of surgery and radiotherapy and a psychiatric one on how patients can be helped to cope with their disease with emphasis on their quality of life.

Many individual minor criticisms of commission or omission might be made but the editors are to be congratulated on their achievement in bringing together the varied authorship into a coherent whole which fills an important niche in the care of patients with cancer. This book has 500 pages in a relatively small print with 1000 words per page. It therefore covers a lot of text! It is well written, referenced and remarkably up to date. At a price of $£ 98$ it probably will not find its way onto every oncologist's bookshelf which is a pity because it makes an excellent reference book. At the very least all departmental libraries should have a copy.

Norman M. Bleehen 